\title{
Providing anti-retroviral therapy in the context of self-perceived stigma: a mixed methods study from Tanzania
}

EDITH A.M. TARIMO ${ }^{1 *}$ and JOHN GEORGE ${ }^{2}$

${ }^{1}$ Muhimbili University of Health and Allied Sciences, P O Box 65004, Dar es Salaam, Tanzania

${ }^{2}$ Family Health International, P O Box 60763, Dar es Salaam, Tanzania

\begin{abstract}
Adherence to anti-retroviral treatment (ART) has been a significant step towards improving quality of life among people living with HIV. However, stigma has been described to influence adherence to ART. A cross-sectional mixed methods study was conducted to explore factors related to stigma and perceived influence of stigma on adherence to treatment amongst ART-prescribed patients and health care providers, respectively in Tanzania. Stigma was assessed through interviewer administered survey among 295 patients. The results from patients showed that 279/295 (95\%) were satisfied with the services provided at the Care and Treatment Centres (CTCS). The set up of CTCS 107/295 (36\%), and queuing at the CTCs $88 / 295$ (30\%) were associated with stigma $(\mathrm{P}<0.001)$. The perceived influence of stigma on adherence to ART was assessed using focus group discussions (FGDs) of 33 health care providers (HCPs). Through FGDs, HCPs perceived the set up of CTCs as friendly yet violated confidentiality. The HCPs reported that ART-prescribed patients hide identifiable cards to avoid being recognised by other people. Some patients were reported to rush to avoid familiar faces, and due to the rush they picked wrong medicines. Also some patients were reported to throw away manufacturers' box with dosage instructions written on the box, resulting in use of doses contrary to the prescriptions. We conclude that despite the fact that most patients were satisfied with the services provided at the CTCs, it is important that HCPs provide dosage instructions on another piece of paper or use disposable bags. A common dispensing window for all patients regardless of the diagnosis may be useful to minimize stigma. Also HCPs may introduce appointment system to avoid long queue at the CTCs.
\end{abstract}

Keywords: Stigma, adherence, HIV/AIDS, ART, Tanzania

\section{Introduction}

Initiation of anti-retroviral therapy (ART) has transformed the perception of AIDS from an epidemic to a manageable chronic disease (Bangsberg et al., 2000). ART use by people living with HIV and AIDS has also decreased transmission of HIV. The sub-Saharan Africa Region which constitutes $69 \%$ of people living with HIV and AIDS globally (UNAIDS, 2010) has received ART coverage with a 20\% increase between 2009 and 2010 alone. As a result, there has been a steady decline in AIDS-related deaths across the region (UNAIDS, 2011). Availability and accessibility of ART is crucial to maximize adherence to treatment among ART-prescribed patients. However, as stipulated in the WHO report, provider and health system-related determinants, which make up the health care environment in which patients receive care, may have a major effect on adherence (WHO, 2003).

Tanzania with an HIV prevalence of $5.1 \%$ among adults aged 15 to 49 years of age (TACAIDS, 2013), started to provide ART to patients with AIDS in October 2004. However, only $30 \%$ of people living with HIV are able to access this important service (UNAIDS, 2010). Although the current national effort is to expand Care and Treatment Centres (CTCS) in regions of greatest need, several factors may influence adherence to treatment among ART-prescribed patients. The WHO report shows that some of the ART-prescribed patients may not take their medicines as prescribed due to socio-economic factors, the health care system, the characteristics of the disease, disease therapies and patient-related factors. Solving the problems related to each of these factors is necessary if patients' adherence to therapies is to

\footnotetext{
* Correspondence: Edith A.M. Tarimo; E-mail:etarimo54@yahoo.co.uk
} 
be improved (WHO, 2003). Poor adherence to ART can lead to the virological failure and spread of multidrug resistant forms of the virus among ART-prescribed patients (Bussmann et al., 2009). Statistics indicate that ART success in Africa Region is a function of patients' desire to fulfil social responsibility (Ware et al., 2009). It is therefore important that long-term adherence must address challenges related to health and life in terms of marginalization and exclusion as a result of stigma (Ware et al., 2006).

Self perceived stigma may hinder adherence to ART. Perceived stigma in the context of ART refers to association of people taking ART with AIDS patients. Previous studies in Africa show that HIV-related stigma hinders access and retention to HIV care services and adherence to ART (Baltazary et al., 2011; Kingori et al., 2012). A multi-site study in five African countries showed that ART-prescribed patients who missed taking their ARV doses had a significant increase in stigma over time (Dlamini et al., 2009). Although HIV-related stigma may be constructed differently, it is expressed in the same pattern across settings (Baltazary et al., 2011; van Tam et al., 2011; Kingori et al., 2012). Also universally, fear of disclosure of HIV status has been reported to influence adherence to ART. People with the latest stage of AIDS are more vulnerable to stigma than those at an asymptomatic stage (Castro \& Farmer, 2005). In most parts of the world, evidence shows that stigma affects adherence among ART-prescribed patients (Wasti et al., 2012). A previous study has shown that ART can be successfully administered within a co-recreational setting; trust and therapeutic relationship between patient and physician in the ART initiation process; characteristics of the therapeutic agents and the degree of social isolation (Altice et al., 2001).

In Tanzania, there is limited knowledge about ART use and issues pertaining to stigma particularly within the health facilities. Knowledge about stigma and delivery of ART at health care facilities can inform planners and hospital administrators of ways to ensure proper set-up of CTCs in order to reduce stigma. Therefore, this study aimed to assess perceived stigma related factors and perceived influence of stigma on adherence to ART among ART-prescribed patients and health care providers respectively.

\section{Materials and Methods}

\section{Study areas}

This cross-sectional study was conducted in 2007 and involved three selected referral hospital namely: Muhimbili National Hospital in Dar es Salaam, Bugando Medical Centre in Mwanza and Kagera Regional Hospital in Bukoba, Tanzania. The hospitals were selected because they serve as referral centres in the respective geographical zones and were among the first facilities to provide ART services in Tanzania.

\section{Quantitative data collection}

An interviewer administered survey was administered to 295 ART-prescribed patients. This sample was calculated using $25 \%$, a proportion of people on ART during the study period. The inclusion criteria among others were ART-prescribed patients who had been on treatment for more than four months at the respective CTCs. The questionnaire was structured with close ended questions to determine the satisfaction with services and self-perceived stigma among ART-prescribed patients.

A simple random sampling to recruit patients into this part of the study was used. All patients who fulfilled inclusion criteria were referred to the research assistants (an intern doctor and an intern nurse) who in turn assigned each patient with a number; those patients who received odd numbers were individually informed about the objectives of the study, and they were requested for consent to participate in the study. 
Consenting patients were asked general questions such as: how did they perceive their health status at the time of data collection, experiences of getting episodes of opportunistic infections, other treatment received besides ART within the last 6 months, the time it took them to seek medical and counselling services when they felt ill, satisfaction with the services at the respective CTCs and about self-perceived stigma.

\section{Qualitative data collection}

The quantitative data was supplemented with qualitative data from the three focus group discussions (FGDs) of 33 health care providers (HCPs). Inclusion criterion was HCPs who had been working full time in the respective CTCs for 10 months or more. The FGD guide was semistructured with open ended questions to explore effects of self-perceived stigma on adherence to ART among ART-prescribed patients as perceived by the HCPs. The FGD guide was constructed in English, translated into Kiswahili (the national language of Tanzania) and back translated. The Kiswahili version was used to moderate the discussion.

Purposive sampling was used to recruit participants to the FGDs. All HCPs who had been providing services at CTCs for one year or more were considered eligible for the study because they had rich information about the study. The participants consisted of a mixture of health care providers; groups ranged between 10 and 13 participants. The primary research question to the HCPs was: "What is the effect of stigma on the adherence to ART among ART-prescribed patients?' Probes were added to the FGD guides to sort for clarifications. During discussion, audio-recorder was used, the first author moderated the discussion, and the co-author took notes in attempt to avoid losing any valid information from the study participants. The FGDs lasted for 60-90 minutes.

\section{Data analysis}

For quantitative data, frequency distribution of all variables was tabulated, and all statements related to stigma and the use of ART was presented in percentages. Cross tabulation was performed between the selected variables. The differences were assessed by Chi-square test. A p-value of less than 0.05 was considered significant. Statistical analysis was conducted using Epi info 6 statistical package.

Qualitative analysis began immediately after data collection, and thematic content approach was used (Krueger, 1998). The two authors listened to the audio tapes to capture the main ideas from the discussion. One of the research assistants transcribed the data word for word and translated the transcripts into English. The first author counterchecked both English and Kiswahili transcripts for translation consistency. Also the first author coded all transcripts manually. The codes were written on the margins of the transcripts, followed by category formation. Both authors discussed the analysis process and preliminary results; minor differences in interpretation were discussed and negotiated for consensus.

\section{Ethical considerations}

Ethical clearance for this study was obtained from Institutional Review Boards of Muhimbili University of Health and Allied Sciences, and Research and Publication Committee of Weil Bugando University College of Health Sciences/Bugando Medical Centre. Permission letters were obtained from Executive Directors of Muhimbili National Hospital and Kagera Regional Medical Officer. Written and oral consents were obtained from ART-prescribed patient and health care providers respectively after explaining the objectives of the study. All health care providers consented for tape-recording of the discussion. During the discussion, anonymity was assured by assigning numbers to each participant. All FGDs participants are referred to by their profession in the results' section. 


\section{Results}

\section{Quantitative survey}

Participants: The quantitative sample consisted of 295 ART-prescribed patients: Muhimbili National Hospital 109 (37\%); Kagera Regional Hospital 99 (34\%) and Bugando Medical Centre 87 (29\%). Most of the patients (66\%) were female and their mean age was 38 (SD 7.9). Majority (85\%) were single and Christians (66\%); attained primary education (65.1\%) and were occupied doing petty business (32.5\%) (Table 1 ).

Table 1: Socio-demographic characteristics of the sample

\begin{tabular}{llll}
\hline Variables & Response & Number & Percent \\
\hline Sex & Female & 194 & 66 \\
Religion & Male & 101 & 34 \\
& Christians & 196 & 66.4 \\
Level of education & Moslems & 95 & 32.2 \\
& Others & 4 & 1.4 \\
& Never attended school & 24 & 8 \\
& Primary School & 192 & 65.1 \\
Occupation & Secondary School & 63 & 21.4 \\
& Technical School & 8 & 2.7 \\
& Higher education & 8 & 2.7 \\
& Business & 96 & 32.5 \\
& Peasant & 82 & 27.8 \\
& Unemployed & 51 & 17.3 \\
& Civil servants & 27 & 9.2 \\
& Private sector & 24 & 8.1 \\
& Students and others & 15 & 5.1 \\
\hline
\end{tabular}

Health status perception of the patients: During the interviews, although most patients ( $n=177$, $60 \%$ ) reported episodes of opportunistic infection ever since they were diagnosed HIV positive, they still perceived their health status as good (72.9\%) (Table 2). Almost half of the patients (145; $49 \%)$ needed treatment beyond ART within the last six months. Overall, 279 (95\%) were satisfied with the service provided at the CTCs.

Table 2: Health status perception of the patients

\begin{tabular}{lll}
\hline Individual's general health status & Frequency & Percent \\
\hline Very bad & 1 & 0.3 \\
Bad & 12 & 4.1 \\
Average & 38 & 12.9 \\
Good & 215 & 72.9 \\
Very good & 29 & 9.8 \\
Total & $\mathbf{2 9 5}$ & $\mathbf{1 0 0}$ \\
\hline
\end{tabular}

Self-perceived stigma amongst ART-prescribed patients: Overall, the levels of self-perceived stigma were low in this study. Minority $(71,23.5 \%)$ of the patients stated that they felt uncomfortable to take medicines (ART) in front of other people. A few patients (48, 16.3\%) avoided or delayed seeking health care for HIV and AIDS because of perceived fear of health care providers' attitude towards their HIV status. Similarly, few patients $(58,19.7 \%)$ delayed treatment seeking because of HIV status (Table 3 ). 
Table 3: Self-perceived stigma among ART- prescribed patients

\begin{tabular}{|c|c|c|}
\hline Variables & Number & Percent \\
\hline $\begin{array}{l}\text { Delaying treatment seeking for medical and counselling } \\
\text { services when felt ill because of HIV status }\end{array}$ & 58 & 19.7 \\
\hline $\begin{array}{l}\text { Experiencing other people explaining something negative } \\
\text { about the clinic [respective CTC] }\end{array}$ & 77 & 26.0 \\
\hline $\begin{array}{l}\text { Feeling bad when people see you [AIDS patients ] queuing } \\
\text { at ART clinic }\end{array}$ & 88 & 29.8 \\
\hline $\begin{array}{l}\text { Ever avoided or delayed seeking health care treatment } \\
\text { because of fear of service providers' attitude towards a } \\
\text { person with AIDS }\end{array}$ & 48 & 16.3 \\
\hline $\begin{array}{l}\text { Uncomfortable taking medicines (ART) from the dispensing } \\
\text { window in front of other people }\end{array}$ & 71 & 23.5 \\
\hline $\begin{array}{l}\text { Uncomfortable with the separation of ART clinic from other } \\
\text { medical clinics at the respective facility }\end{array}$ & 119 & 40 \\
\hline Set-up of the CTCs breaching confidentiality & 107 & 36.3 \\
\hline $\begin{array}{l}\text { Ever went to a health facility that is far away, instead of } \\
\text { going to a nearby facility because of HIV status }\end{array}$ & 53 & 17.9 \\
\hline
\end{tabular}

Self-perceived stigma by sex: A total of 88 patients responded to the question on self-perceived stigma. Significantly more males (38.6\%) than females (25.3\%) felt bad queuing at the CTCS $(p<0.01)$. Also significantly more males $(23.8 \%)$ than females $(17.9 \%)$ ever went to a health facility that is far away, instead of going to a nearby facility because of HIV status $(p<0.05)$.

Self-perceived stigma by health facilities as perceived by patients: Comparing with the other two hospitals, most of the patients (50.6) in Bugando Medical Centre felt uncomfortable queuing to get services at the CTCs. In the same hospital, the majority of the patients (54\%) reported that the set up of the CTC could breach confidentiality, and that most (42.5\%) patients took time to seek health care services compared to other regions. Patients attending Bugando Medical Centre perceived the services to be poorer than in the other hospitals. The major reason for delaying seeking health care services was fear of being seen by other people (Table 4).

Table 4: Self-perceived stigma among ART-prescribed patients by hospital

\begin{tabular}{|c|c|c|c|c|}
\hline Factors & Muhimbili & Bugando & Kagera & Total (Percent) \\
\hline Felt bad queuing at the CTC & $28(25.7 \%)$ & $44(50.6 \%)$ & $16(16.2 \%)$ & $88(29.8 \%) * * *$ \\
\hline $\begin{array}{l}\text { CTC set-up breaches } \\
\text { confidentiality }\end{array}$ & $43(39.4 \%)$ & $47(54.0 \%)$ & $17(17.2 \%)$ & $107(36.3 \%) * * *$ \\
\hline $\begin{array}{l}\text { Delaying treatment seeking } \\
\text { for medical and counselling } \\
\text { services when felt ill because } \\
\text { of HIV status }\end{array}$ & $9(8.3 \%)$ & $37(42.5 \%)$ & $12(12.1 \%)$ & $58(19.7 \%) * * *$ \\
\hline
\end{tabular}

\section{Qualitative Results}

The FGDs consisted of 33 participants: eight physicians, 17 nurse/counsellors, three pharmacists, three laboratory technologists and two social workers. All participants were aged above 18 years and were full time workers at the respective CTCs. The FGD findings highlighted what the HCPs perceived as the effect of self-perceived stigma to ART adherence among ART-prescribed patients. To facilitate understanding of the qualitative dataset, two themes emerged. The first theme, 'HCPs' perceptions about comfort at CTCS amongst ART- prescribed patients' underscores the positive aspects of the CTCs' environment. The second theme, 'HCPs 
perceptions about discrepancies at CTCS and ART use' addresses the negative aspects of the CTCs' environment. The second theme is divided into categories as shown in the subsequent sections.

HCPs' perceptions about comfort at CTCS amongst ART- prescribed patients: The HCPs described the set-up of CTCS as friendly, and they believed that most ART-prescribed patients appreciated the quality of services and were passing on positive messages about the clinic to the surrounding community. One of the HCPs quoted a patient saying: "Those who get service here $[\mathrm{CTC}]$ do invite their friends from the village to get similar treatment... After availability of the treatment, HIV is no longer a problem here" (Participant 3, a nurse/counsellor, Kagera Regional Hospital).

Most HCPs perceived the CTCs' set-up positively because they saw patients sharing their experiences while waiting for the services. They witnessed some patients encouraging in dialogue and giving advice to each other; how to eat well and how to use treatment correctly. Thus, the HCPs stated that patients used CTCs as comfortable meeting places: "They encourage each other; they advise each other ... They are used to this place to the extent that when they are in groups, they discuss two or three issues ...: how to eat; experiences of using the medicines and general progress of their health status" (Participant 1, a medical doctor, Muhimbili National Hospital). They added that through the clinic, some patients became friends and visit each other. Nevertheless, the HCPs noted that some patients preferred one clinic over others; consequently this preference interfered with the continuity of their medicines: "Other patients may come ..., and we may register them thinking that they are new patients at this place. After registration, they tell you [HCP], 'is better I have transferred myself here; I shall tear up the card from the other place...' That means they had been at treatment elsewhere!"' (Participant 6, a nurse/counsellor, Bugando Medical Centre).

HCPs' perceptions about discrepancies at CTCs and ART use: Despite the perceived friendly nature of CTCs' set-up, the HCPs stated that some of the ART-prescribed patients were uncomfortable at CTCs. They reasoned that these patients were not ready to disclose their HIV status to the surrounding community. Therefore, they believed that the separation of the CTCS from other clinics disclosed the patients' status to people who were not connected to the clinic, and hence exposed them to stigma. They said: "Many people know this corridor [CTC]; the corridor [CTC] serves patients with AIDS... visitors may be interested to differentiate patients with AIDS from other patients. Other clients may stare at those on ART; '...despite his/her healthy looking status, is he/she infected as well?'... When patients with cardiac problems say that, those on ART feel stigmatized" (Participant 6, a medical doctor, Muhimbili National Hospital).

Other HCPs emphasised that sharing of the CTCs' corridor between AIDS patients and clients with other medical conditions was not user friendly. They said that ART-prescribed patients were loudly called by names from the pool of clients to collect medicines at the ART dispensing window. The HCPs observed that most patients were uncomfortable with this procedure. Several HCPs said they witnessed patients striving to escape when they were called to collect medicines. By so doing some patients left without collecting their medicines. They said: "... Others may just leave or may come to knock at my [doctor] door requesting to get in; 'Sorry doctor ... I saw my neighbour right there; I cannot take medicines from that window!" (Participant 1, a medical doctor, Muhimbili National Hospital). A pharmacist commented that when he called patients by names, some of them reacted negatively. He said that at one point, he was confused with the patients' reactions. One nurse complemented: "When patients arrive, I call them by their names... One of the patients became furious. I thought he/she was very ill; I called him/her into my room to explore what was wrong. He/she told me, 'I saw my colleague out there ...; I don't want him/her to know that I receive service here. Now, I want to leave and sit somewhere else..." (Participant 9, nurse/counsellor, Muhimbili National Hospital). Other HCPs 
discussed how they dealt with similar challenges. They said they tried to lower the voice when they called patients for various services. However, they realized that lowering the voice was not the best. They recalled other clients around the hospital commenting, 'Why should they be called in low voice?' Therefore, they said they decided to continue calling the patients in normal volume.

The HCPs realized that patients with high socio-economic status were not ready to be seen around the CTCs. They said, without involving other health care providers, these patients were making direct appointments with physicians. As a result, when additional service such as tuberculosis test was demanded, they observed that most of these patients were not seeking such services from the respective HCPs. In addition, they noted that these patients were coming late and sometimes they missed important services: "Sometimes they [ART-prescribed patients] come very late when the laboratory technician had left. You know sometimes I may need to check their CD4 counts; definitely they will miss that... that is a loss... they will have to come back to get the test" (Participant 7, a medical doctor, Bugando Medical Centre).

The HCPs elaborated that people who were perceived with high socio-economic status such as doctors, teachers, district officials, and members of the parliament were the most self stigmatizing people. The HCPs said that such people feared distorting their social status by showing up at the CTCs. They supported the argument that the set-up of the CTCs may contribute to stigma: "People look at CTC from a distance and ask, "why should I enter that building? People may think that I am going to get ART there!' ... people tend to look back and forth to avoid familiar faces.... the clinic is well known that it serves people with AIDS" (Participant 7, a pharmacist, Kagera Regional Hospital).

Despite the HCPs' effort to encourage all people regardless of their social status to come to the CTCs for various services, they observed that most people including HCPs were not ready to seek services at CTCs. One HCP said: "The main issue is when you talk about professionals; they have their prominent positions. I mean highly prominent people more experience self stigma than ordinary people. They want to protect their identity... these are doctors, nurses, teachers, businessmen, educated leaders, members of the parliament, district commissioners and others. So in the era of stigma, we [HCPs] are not exceptional" (Participant 4, nurse/counsellor, Kagera Regional Hospital). The HCPs also said that queuing for services at CTCs contributed to stigma. One participant was offended by the queue. He /she said: "That queue starts there [pointing at a site where queue starts] and it is notable. When I was passing there this morning, I heard people commenting, 'Those are HIV infected; they arrive at 6 in the morning; they line-up'. To me, that is painful” (Participant 6, a lab technician, Kagera Regional Hospital).

Effect of stigma on the correct use of ART: The HCPs reported that, at the CTCs, ARTprescribed patients were always rushing to avoid familiar faces. They said that patients looked confused at the pharmacy particularly when they heard similar names, and this lead some of them to collect incorrect medicines. One participant admitted: "It has happened many times; some patients' names are similar. Or if they hear you [pharmacist] wrongly, they may come closer to the window, collect medicines and leave while the medicines belong to another patient ... We have been following up with such patients several times... we have been calling them to bring back the medicines. Some of the patients may stay home with incorrect medicines for two days before bringing them back!" (Participant 8, a pharmacist, Muhimbili National Hospital). Another participant added: "Even yesterday, one patient brought back the medicines; different medicines from the prescription in is his file... he had already used the medicines with a notion that he takes medicines in the morning and evening...he took medicines that were supposed to be taken once in the evening...; the medicines affected him." (Participant 5, a nurse/counsellor Muhimbili National Hospital). 
On the contrary, the HCPs noted that literate patients knew their medicines and they were likely to use the medicines correctly: "When he [literate ART-prescribed patient] is given different medicines, he tells you, "I have been on medicines named Niverapine. Why should I get Efavirenz today? Why am I given incorrect medicines?” (Participant 6, a medical doctor, Muhimbili National Hospital).

Contribution of placement of dosage instructions to stigma: The HCPs noted that, medicine dispensers were writing the dosage instructions on the manufacturers' box. They realized that some patients took the medicines out of the boxes immediately after they left the pharmacy. Most of the HCPs said they witnessed a lot of empty boxes scattered in the toilets, waiting room/reception, and other places around the hospital. From their experience they believed that ART-prescribed patients were not taking the boxes home because of fear of disclosing their HIV status to the family members who could associate the boxes with their HIV status. Given that dosage instructions were written on the boxes, most HCPs were worried that patients might have been using their medicines incorrectly. One HCP contextualized the situation: "When they leave the clinic..., they take out the containers [container with medicines] and threw away the boxes. That means they do not take the boxes home. So perhaps they may use the medicines against the instructions" (Participant 2, nurse/counsellor, Muhimbili National Hospital).

Other HCPs stated that they came across ART-prescribed patients who presented with side effects associated with misuse of medicines. They said that they were shocked when patients presented with side effects two days after taking medicines, 'I cannot even lift up my eye lids', one patient complained to the doctor. When they [doctors] asked those patients to bring back the medicines which they had been taking, surprisingly the patients brought the containers without the boxes. To make the situation worse, one doctor noted that the patient re-wrote the instructions on the containers incorrectly. He narrated: "Yesterday a patient came with a container and the dosage was written, 'one Efavirenz twice'. I asked him/her, 'who wrote this [dose on the container]', he said, 'myself!' I asked where the box is. He/she said 'I took the container out of the box...' when you walk around the building there is plenty of boxes scattered here and there...; in the toilets, there are many empty boxes" (Participant 1, a medical doctor, Muhimbili National Hospital).

Use of identifying cards among ART-prescribed patients accelerates stigma: The HCPs explained the discrepancy of using blue cards among ART-prescribed patients. They said that most ARTprescribed patients were hiding their cards because of fear of disclosing their status through those cards. They reasoned that people were likely to differentiate ART-prescribed patients from others by the colour of cards. They said: "People know those blue cards; when you ask them [ART-prescribed patients] to sit on the benches so that you can serve one by one, many people [non-ART prescribed patients] may come closer to observe what is being given to the one with the blue card. You may force them to leave, but they will strive to come closer ...they pay attention until when the patient [ART-prescribed patient] leaves ..." (Participant 8, a pharmacist, Muhimbili National Hospital). Another HCP supported the discourse as follows: "Often, I have observed people; when you call a patient, another person may closely look at him/her ... So they [non-ART users] stare at them [ART-prescribed patients]" (Participant 10, nurse/counsellor, Muhimbili National Hospital).

The HCPs noted that majority of people were aware of the 'blue cards' and the users. For such a reason, patients were hesitating to hold their cards openly while sitting in the waiting room. As a result, some patients were deciding to leave without picking their medicines. Sometimes they relocate themselves to other CTCS to maintain their privacy. Several HCPs 
reported to see patients going secretly to the CTCs they preferred while hiding the 'blue cards', and those were hospital workers and well known people in the community.

\section{Comparison of the quantitative and qualitative results}

The ART-prescribed patients' experiences correspond to the HCPs' observations in the following aspects: The low magnitude of self-perceived stigma amongst ART-prescribed patients support the observations made by HCPs. The HCPs noted that the CTCs were comfortable places for patients to share their experiences. On the other hand, the HCPs observed discrepancies in the CTCs. These discrepancies support the presence of negative aspects towards CTCs' albeit low in magnitude as perceived by ART-prescribed patients. Delaying treatment seeking among patients may be associated with fear of disclosure of their HIV status to the surrounding community.

\section{Discussion}

Overall, the results indicate that ART-prescribed patients experience low magnitude of selfperceived stigma at CTCs. However, the fact that the set-up of the CTCS contributes to stigma and that self-perceived stigma influence adherence to treatment among ART-prescribed patients is worth noting. From the quantitative results, the ART-prescribed patients felt uncomfortable to take medicines in front of other people suggesting that patients do not want to disclose their HIV status to other people. This is evidenced along the behaviour of delaying to seek medical treatment and feeling bad to queue at CTCs. From the qualitative findings, the HCPs perceived the CTCS as comfortable places for the patients to share their experiences and concerns. However, the negative aspects that were brought up by HCPs increase the readers' understanding of the patients' responses in the quantitative results. For example, in this context, the behaviour of ART-prescribed patients hiding identifiable blue cards and throwing away the identifiable manufacturers' boxes with dosage instructions might have been fuelled by stigma among ART-prescribed patients. In addition, the rush to avoid familiar faces that led to collection of incorrect medicines from the dispensing window is facilitated by fear of stigma.

The perception that the CTCs' environment breaches confidentiality implies that ARTprescribed patients may deter to seek services from the respective CTCs. In principle, like any other medical condition, ART-prescribed patients deserve rights to confidentiality of their health status. In Zambia, lack of confidentiality in the treatment centres was identified as barrier to patients' adherence to antiretroviral treatment (Sanjobo et al., 2008). Thus, when physical set-up of health facilities breaches the confidentiality, most patients are unlikely to use the clinic and consequently adhere to the treatment. In Thailand, health care providers were encouraged to provide people living with AIDS with more knowledge on HIV/AIDS and ART use (Chamroonsawasdi et al., 2011). Therefore, it is important that ART-prescribed patients get adequate knowledge and experience user friendly services to facilitate adherence to treatment. In the present study, the demand for user friendly service among ART-prescribed patients at CTCs may necessitate hospital planners and administrators to integrate the CTCs' services within the existing health care facilities.

In this study, self-perceived stigma emerges as a potential barrier to ART adherence among ART-prescribed patients. The rush to avoid familiar faces among ART-prescribed patients may lead to missed doses. A previous multi-country study carried out in Lesotho, Malawi, South Africa, Swaziland, and Tanzania indicated the consequences of stigma on medicine intake; stigma increased the frequency of missing medications among HIV positive people (Dlamini et al., 2009). Similarly, in Kenya, patients who reported experiencing stigma were those who related to other people's attitudes towards their HIV status, and these patients 
were likely to not adhere to the prescribed medicines (Kingori et al., 2012). Although much has been documented to increase community awareness about HIV/AIDS and stigma reduction, evidence suggests that more effort is needed to overcome the impact of stigma among ARTprescribed patients. In this study, the effects of stigma contextualize itself within the individuals, communities and the CTC surroundings. Similarly, a previous study in Tanzania revealed that because of stigma, patients with AIDS changed their behaviour to avoid negative reactions towards them, both from health facilities and communities (Mutalemwa et al., 2008).

Moreover, the struggle described by the HCPs about ART-prescribed patients trying to hide identifiable cards, indicates the patients' efforts of consoling and fitting in the group of HIV negative people. This kind of behaviour is supported by Goffman (1963). Goffman (1963) believes that when an individual comes in contact with other people, that individual will attempt to control or guide the impression that others might make of him by changing or fixing his or her setting, appearance and manner. Similarly, Parker \& Aggleton (2003) conceptualize that stigma is linked to the reproduction of social differences. In Nigeria, some patients were not fully adhering to their ART regimen because of fear of social rejection (Uzochukwu et al., 2009). Similarly, in Kenya, the fight against stigma has been a government and nongovernmental organization's priority, where health education and counselling services have been found helpful to combat stigma (Odindo \& Mwanthi, 2008).

This study had a number of limitations. The data were collected in 2007. Taking into account the current progress in ART delivery, changes to allay the reported discrepancies may have already taken place in the respective facilities. However, the presented data still make sense given the ongoing documentations about stigma and ART use in the literature. Also the use of mixed methods increases our understanding of the results.

Confidentiality being one of the fundamental rights of clients regardless of their medical condition should be emphasised when designing health facilities and the mode of delivering the services should be improved. It may be important to design less identifiable cards for the ARTprescribed patients in order to eliminate obvious distinctions among clinic attendees. Appropriate planning of CTCs placement/arrangement in line with other clinics is crucial to improve patients' comfort and access to basic health care package including user-friendly clinic. Although all ART-prescribed patients are provided with routine supportive counselling on medications use and laboratory monitoring for the effects of the prescribed medicines, patients need consistent education to overcome stigma. Further studies are recommended to identify specific reasons why stigma factors rank higher in Bugando than in other facilities.

In conclusion, since self-perceived stigma may hinder use of CTCS and adherence to treatment amongst ART-prescribed patients. Planners and administrators of facilities are called upon to ensure that all clients experience user friendly services regardless of their health status. Also the findings of this study call for all health care providers to be more self-aware and use the power they have to monitor their own actions that may increase stigma. HCPs should develop mechanisms such as writing dosage instructions on a separate piece of paper or use disposable bags; introduce appointment system to avoid long queue and use of a common dispensing window for all patients regardless of their medical conditions. This may ensure that self-perceived stigma does not affect adherence to ART amongst ART prescribed patients. It is therefore necessary to implement such simple interventions to address stigma and ensure adherence amongst ART-prescribed patients. 


\section{Acknowledgements}

The authors would like to acknowledge the study participants for consenting to participate in the study and Emanuel Mpolya and Emanuel Maganga for recruiting and interviewing study participants. Dr. Anne Outwater is acknowledged for her contribution in editing the manuscript. The study was funded by the Swedish International Development Agency through a bilateral research capacity building grant to the Muhimbili University of Health and Allied Sciences, Tanzania.

\section{Conflict of interest}

Authors declare no conflict of interest in relation to this study

\section{References}

Altice, F.L., Mostashari, F. \& Friedland, G.H. (2001) Trust and the acceptance of and adherence to antiretroviral therapy. Journal of Acquired Immune Deficiency Syndromes 28, 47-58.

Baltazary, G., Akarro, R. R., \& Mussa, A. S. (2011) Some factors associated with non-adherence to antiretroviral therapy (ART) in people living with HIV/AIDS (PLHA) in Tanzania: a case study of Muhimbili National Hospital region. East African Journal of Public Health 8, 237246.

Bangsberg, D. R., Hecht, F. M., Charlebois, E. D., Zolopa, A. R., Holodniy, M., Sheiner, L., Bambergerh, J.D., Chesneyd, M.A. \& Mosse, A. (2000) Adherence to protease inhibitors, HIV-1 viral load, and development of drug resistance in an indigent population. AIDS 14, 357-366.

Bussmann, H., Wester, C.W., Thomas, A., Novitsky, V., Okezie, R., Muzenda, T., Gaolathe, T., Ndwapi, N., Mawoko, N., Widenfelt, E., Moyo, S., Musonda, R., Mine, M., Makhema, J., Moffat, H., Essex, M., DeGruttola, V. \& Marlink, R. (2009) Response to zidovudine/didanosine-containing combination antiretroviral therapy among HIV-1 subtype C-infected adults in Botswana: two-year outcomes from a randomized clinical trial. Journal of Acquired Immune Deficiency Syndromes 51, 37-46.

Castro, A., \& Farmer, P. (2005) Understanding and addressing AIDS-related stigma: from anthropological theory to clinical practice in Haiti. American Journal of Public Health, 95, 53-59.

Chamroonsawasdi, K., Insri, N. \& Pitikultang, S. (2011) Predictive factors of antiretroviral (ARV) drug adherence among people living with HIV/AIDS attending at Taksin Hospital, Bangkok, Thailand. Journal of the Medical Association of Thailand 94, 775-781.

Dlamini, P.S., Wantland D., Makoae, L.N., Chirwa, M.,Kohi, T.W., Greeff, M., Naidoo, J., Mulla, J., Uys, L.R. \& Holzemer, W.L. (2009) HIV stigma and missed medications in HIV-positive people in five African countries. AIDS Patient Care STDS 23, 377-387.

Goffman, E. (1963) Stigma: Notes on the management of spoiled identity. New York: Simon \& Schuster Inc.

Kingori, C., Reece, M., Obeng, S., Murray, M., Shacham, E., Dodge, B., Akach, E., Ngatia, P. \& Ojakaa, D. (2012) Impact of internalized stigma on HIV prevention behaviors among HIVinfected individuals seeking HIV care in Kenya. AIDS Patient Care STDS 26, 761-768.

Krueger, R. A. (1998) Analysing and Reporting Focus Group Results: Focus Group Kit 6. Thousands Oaks: Sage Publications. 
Mutalemwa, P., Kisoka, W., Nyigo, V., Barongo, V., Maalecela, M. V. \& Kisinza, W.N. (2008). Manifestations and reduction strategies of stigma and discrimination on people living with HIV/AIDS in Tanzania. Tanzania Journal of Health Research 10, 220-225.

Odindo, M.A. \& Mwanthi, M.A. (2008) Role of governmental and non-governmental organizations in mitigation of stigma and discrimination among HIV/AIDS persons in Kibera, Kenya. East African Journal of Public Health 5, 1-5.

Parker, R. \& Aggleton, P. (2003) HIV and AIDS-related stigma and discrimination: a conceptual framework and implications for action. Social Science \& Medicine 57, $13-24$.

Sanjobo, N., Frich, J.C. \& Fretheim, A. (2008) Barriers and facilitators to patients' adherence to antiretroviral treatment in Zambia: a qualitative study. SAHARA Journal 5, 136-143.

THMIS (2013)Tanzania HIV/AIDS and Malaria Indicator Survey 2011-12. TACAIDS, ZAC, NBS, OCGS and ICF International, March 2013.

UNAIDS Global Report. Geneva: http://www.unaids.org/globalreport/documents/20101123_GlobalReport_full_en.pdf (Accessed 15/6/2012).

UNAIDS (2011) 2011 UNAIDS World AIDS Day Report. UNAIDS, Geneva

Uzochukwu, B.S., Onwujekwe, O.E., Onoka, A.C., Okoli, C., Uguru, N.P. \& Chukwuogo, O.I. (2009) Determinants of non-adherence to subsidized anti-retroviral treatment in southeast Nigeria. Health Policy and Planning 24, 189-196.

Van Tam, V., Pharris, A., Thorson, A., Alfven, T. \& Larsson, M. (2011) "It is not that I forget, it's just that I don't want other people to know": barriers to and strategies for adherence to antiretroviral therapy among HIV patients in Northern Vietnam. AIDS Care 23, $139-145$.

Ware, N.C., Idoko, J., Kaaya, S., Biraro, I.A., Wyatt, M.A., Agbaji, O., Chalaamilla, G. \& Bangsberg, D.R. (2009) Explaining adherence success in sub-Saharan Africa: an ethnographic study. PLoS Medicine 6(1), e11.

Ware, N.C., Wyatt, M.A. \& Tugenberg, T. (2006) Social relationships, stigma and adherence to antiretroviral therapy for HIV/AIDS. AIDS Care 18, 904-910.

Wasti, S.P., van Teijlingen, E., Simkhada, P., Randall, J., Baxter, S., Kirkpatrick, P. \& Gc, V.S. (2012). Factors influencing adherence to antiretroviral treatment in Asian developing countries: a systematic review. Tropical Medicine \& International Health 17, 71-81.

WHO. (2003). Adherence To Long-Term Therapies: Evidence for Action. World Health Organization, Geneva. 